\title{
Identidad cristiana y racionalidad de la pertinencia religiosa: su incidencia en las actuales sociedades plurales*
}

\author{
Arturo Calvo Espiga" \\ Recibido: 27 de septiembre de 2013 • Revisado: 18 de noviembre de 2013
}

- Aprobado: 20 de noviembre de 2013

\section{Resumen}

La problemática jurídica implicada en la relación entre identidad y pertenencia, contemplada desde el ámbito de unas culturas diversas que se interrelacionan podría formularse y sintetizarse a través de las cuestiones suscitadas en torno a la validez universal de los derechos humanos y al imperio de la libertad y la racionalidad en las relaciones sociales. El Cristianismo, por la exigencia a sus seguidores de libertad y racionalidad, en su pertenencia religiosa, se asume como referente histórico del análisis de la problemática suscitada en los Estados democráticos a la hora de regular la protección de los derechos relacionados con el respeto de las identidades personales y colectivas y la observancia de los principios democráticos fundamentales en los ámbitos sociales en que se expresan.

Palabras clave: libertad religiosa, derechos humanos, multiculturalismo, pluralismo, democracia.

*Artículo de Investigación. Estudio realizado con la ayuda del Centro de Estudios anejo a la Iglesia Nacional Española en Roma. Facultad de Derecho, Universidad de Málaga, España

*** Licenciado y Doctor en Derecho Canónico, Licenciado y Doctor en Derecho, Licenciado y Doctor en Diritto Civile, Catedrático de Derecho Eclesiástico, Abogado del Tribunal de la Rota de Roma, Facultad de Derecho, Universidad de Málaga, España. Correo electrónico: acalvo@uma.es 


\title{
Christian Identity And Rationality Of The Religious ASCRIPTION: IMPACT ON CURRENT PLURAL SOCIETIES.
}

\begin{abstract}
The juridical problematic involved in the relation between identity and ascription contemplated from the field of some diverse cultures, that interrelate well in the same geographic space or through the universe of the communication, could formulate from the questions aroused around the universal validity of the human rights and to the empire of the freedom and rationality in the social relations. The Christianity, by the exigency to his followers of freedom and rationality in his religious adscription assumes like historical referent of the analysis of the problematic aroused in the democratic States to the hour to regulate the protection of the rights related with the respect of the personal and collective identities and the observance of the fundamental democratic principles in the social fields in that those express.
\end{abstract} Keywords: Religious freedom, human rights, multiculturalism, pluralism, democracy.

\section{IDENTIDADE CRISTÃ E FUNDAMENTAÇÃO DA RELIGIOSA: SEU IMPACTO NA CORRENTE PLURAL SOCIEDADES}

\section{Resumo}

As questões jurídicas envolvidas na relação entre identidade e de pertença, como visto a partir do campo sobre as diferentes culturas que se inter-relacionam poderia ser formulada e sintetizado a partir das questões levantadas sobre a validade universal dos direitos humanos e do Estado de liberdade e racionalidade na relações sociais. Cristianismo, exigindo seus seguidores à liberdade e racionalidade na filiação religiosa assume-se como a análise histórica sobre os problemas levantados em estados democráticos ao regulamentar a proteção dos direitos relativos a respeito das identidades pessoais e grupo e respeito dos princípios democráticos fundamentais nesses ambientes em que são expressas.

Palavras-chave: Liberdade deligiosa, direitos humanos, multiculturalismo, pluralismo, democracia. 


\section{Introducción}

La superficialidad que en sociedades como, por ejemplo, la española, se ha convertido en compañía necesaria de la simplicidad reductora de realidades o fenómenos tan complejos como el del hecho religioso; constituye, sin duda alguna, precioso acicate para urgir nuevos planteamientos y más atentas reflexiones desde el amplio espectro de las ciencias humanas, sociales y jurídicas, sobre el papel y función que la religión y la religiosidad desempeñan en las, al menos teóricamente, laicas y secularizadas sociedades contemporáneas (Bachelard, 1949, p. 148). La presencia de la religión, en sus más diversas y multiformes manifestaciones, se impone como una realidad, como un factum social que por mucho que se intente ocultar, siempre asomará o rebrotará incluso por grietas y resquicios inesperados. Desde el entorno que determinan y en el que a la vez se insertan los actuales modelos de sociedad, intentamos en estas líneas fijar nuestra atención en la incidencia que la identidad personal y la pertenencia social, en su más amplia dimensión, tienen en la configuración y el funcionamiento de las modernas sociedades complejas caracterizadas por una gran novedad respecto a momentos históricos del pasado y su pluralidad religiosa, ideológica, étnica y cultural (Gurevich, 1997, p. 82).

Tampoco hemos de perder de vista que la posición de la persona en la sociedad, así como el desarrollo de su personalidad, viene, en buena medida, definida y regulada por el derecho vigente en tal sociedad. O dicho de otro modo, la posición real de la persona en los ámbitos sociales en que se desenvuelve se refleja en las normas jurídicas y en la interpretación y la aplicación que hace de las mismas. Se puede afirmar que en la relación que mantiene una determinada sociedad respecto al derecho, se manifiesta también la estima que le merece el individuo: el desprecio del derecho o su reducción a un papel irrelevante en el sistema de relaciones sociales implican necesariamente, la violación de los derechos más elementales de los miembros de la sociedad; por el contrario, un alto aprecio del derecho es señal inequívoca de la existencia y la actualización de garantías que protegen la vida humana y que son tenidas en cuenta y observadas por la sociedad (Gurevich, 1972, p. 163).

\section{Una cuestión propedéutica}

En toda esta reflexión se ha de tener muy presente que la identidad y la pertenencia son dos realidades dialécticamente necesarias, puesto que mutuamente se 
complican y simultáneamente se autoexcluyen, porque cuanto más fuerte y comprometida es la pertenencia social con mayor intensidad se obscurece la identidad individual, ya que, como se suele poner de relieve, pertenencia es asimilable a identidad colectiva; aunque por otra parte, solo en un ámbito relacional interpersonal, necesariamente implicado en la pertenencia, es posible la conformación y la consolidación de la propia identidad o personalidad ${ }^{1}$. Pertenecer implica, siempre y de manera obligada, compartir un propósito con otros, es decir, relacionarse con una colectividad que, a la vez, uniforma e identifica o, dicho con mayor precisión, identifica uniformando. El ser humano, desde la más profunda y radical intimidad de su propia naturaleza, de su mismo origen, funda su identidad personal en la pertenencia a una familia, cuya única razón de ser, en cuanto a familia, es posibilitar un sistema de relaciones en el que los individuos que en ella se relacionan maduren progresivamente su identidad frente a la inicial y requerida pertenencia en que se funda y de la que recibe su sentido más profundo (Barcellona, 2006, p. 24-25). En su fundamento original el ser humano se reconoce como relación-pertenenciarelación. La relación padre-madre posibilita biológica y afectivamente el nacimiento del ser humano que, desde el principio, pertenece a esa relación con y en la que, por otra parte, se relaciona esencialmente.

Ahora bien, si tendemos a proteger, fomentar y profundizar nuestra identidad personal y para garantizar nuestra propia personalidad necesitamos de forma decisiva, la relación con una identidad colectiva o la pertenencia a un entramado social que nos sobrepase y comprenda, no cabe la menor duda de que la relación existente entre la identidad individual y la identidad colectiva o pertenencia, conforma y delimita uno de los problemas más urgentes al que han de responder nuestras sociedades pluralistas. Cuestionamiento que, además, afecta de lleno algunos de los asuntos más candentes del ámbito jurídico. Me refiero, por ejemplo, al problema de la validez universal de los derechos humanos, por ser, entre otros, uno de los interrogantes

\footnotetext{
${ }^{1}$ Piénsese, por ejemplo, en una de las modalidades de pertenencia más radicalizada y universalizada en nuestro entorno social: la de los hinchas o forofos de los equipos deportivos, de forma especial por lo que se refiere al futbol. Cuanto más fuerte e intensa es la pertenencia, mayor es la masificación. El individuo se esconde y diluye de tal modo en su pertenencia que es capaz de realizar acciones contrarias y opuestas a sus más íntimas convicciones personales; al tiempo que ve confirmados y aumentados su ego y autoestima cuando el club al que pertenece y con el que, en consecuencia, se identifica, vence un partido comprometido o consigue cualquier campeonato. Nos hallamos en este caso ante un supuesto o modo de pertenencia, en cierta forma paradigmático, que en determinados momentos, propicia e incluso justifica la inversión de valores personales y morales fundamentales: el forofo, por ejemplo, siempre anhela y desea la victoria de su equipo aunque sea consecuencia de flagrante injusticia.
} 
directamente relacionados con la opción o adscripción religiosa de los individuos. En este supuesto, los límites estarían determinados por la pertenencia (identidad colectiva) a un preciso y concreto sistema de valores, tradiciones, religión y cultura; y a la asunción de una categoría estrictamente personal e individual, en razón del sujeto titular de los mismos, como la de los derechos humanos. La problemática jurídica implicada en la relación entre identidad y pertenencia contemplada desde el ámbito de unas culturas diversas, que se interrelacionan bien en el mismo espacio geográfico o bien a través del universo de la comunicación, podría formularse y sintetizarse en la siguiente pregunta: ¿cabe la posibilidad de defender hoy la existencia de derechos humanos válidos universalmente para cada individuo ante la existencia de pertenencias o identidades colectivas diferentes y ajenas a los ámbitos ideológicos, culturales y jurídicos en que aquellos se formularon?

La circunstancia de vivir en un mundo que se ha hecho único (globalizado) nos acucia y obliga a hablar y discurrir sobre problemas que, aunque de necesaria concreción práctica, no han alcanzado todavía la madurez teórica que requiere su comúnmente aceptada y pacífica puesta en práctica². La falta de claridad y comprensión conceptual aboca a errores e imprecisiones que acaban por generar más problemas complejos de los que aparenta solucionar. Error que ha llevado a políticos, intelectuales, ideólogos, e incluso, economistas, a interpretar o explicar los conflictos violentos y las atrocidades que hemos vivido y seguimos padeciendo como un simple corolario de la diversidad y de las divisiones religiosas y culturales. Mas, para amplios sectores de nuestra sociedad, aunque solo sea de manera implícita pero, lógicamente, influyente, el mundo es considerado una especie de federación de religiones y civilizaciones. Ignoran y hacen tabla rasa con este modo de pensar, de la abundancia, riqueza y variado cromatismo de matices con que las personas se perciben así mismas, y a la vez, explicitan, por la irracionalidad de sus planteamientos, la complejidad del sistema de relaciones personales al interior de cada cultura (Maffettone, 2006, pp. 79-82). Propuesta que, con toda probabilidad,

\footnotetext{
${ }^{2}$ Globalización que, al menos en sus constitutivos esenciales y salvando siempre las peculiaridades de nuestro siglo, no constituye un fenómeno novedoso en la historia humana. Como ha recordado un conocido historiador contemporáneo: el propio mundo era concebido por los hombres del Medievo como una unidad y en consecuencia, todas y cada una de sus partes eran percibidas no como autónomas sino como fragmentos de esta globalidad. Todo lo que existe se refiere a un principio regulador central, se inserta en una jerarquía eurítmica y se encuentra y reconoce en relación armónica con los demás elemento del cosmos (...)La esencia moral (...) de la percepción medieval del mundo es también manifestación de su unidad y de su afinidad interior (Gurevich, 1972, pp. 303-304).
} 
parte del prejuicio de que los ciudadanos pueden ser clasificados de forma unívoca según un método singular, único, uniforme y omnicomprensivo ${ }^{3}$. Reduccionismo que empobrece, empequeñece y castra la rica y plural diversidad identitaria del ser humano en sus pertenencias sociales, ideológicas, políticas, culturales y religiosas, sobre todo si se tiene en cuenta que la simplificación, objetivación e imposición de unas u otras pertenencias es incontestable indicio de totalitarismo (Sen, 2006, p. 29).

\section{Razonabilidad de la fe y pertenencia religiosa}

La irrupción del Cristianismo en la consolidada seguridad de las estructuras socio-políticas del Imperio romano provocó una profunda crisis en la vivencia y aceptación de los propios fundamentos del fenómeno de la pertenencia social y política de los individuos, puesto que la identidad de los cristianos, fieles ciudadanos o moradores del Imperio, principalmente venía referida con su pertenencia a una comunidad de fe y solo, de manera secundaria, lo social y lo político determinaban relacionalmente su identidad. Pertenencia que, en el caso de los primeros cristianos provenientes del judaísmo y de la cultura helenística en su mayor parte, les descubrió unas dimensiones de la libertad personal, y este redimensionamiento cristiano de la libertad, consecuencia de su pertenencia a una específica comunidad de fe, aportó también a la novedad de aquellas primeras comunidades, un sentido del todo particular, respecto a otras creencias o ideologías, de lo que significa la pérdida martirial de la vida, es decir, la aceptación racional y razonable de la muerte como testimonio de la propia fe (Braun, 1977, pp. 599-623 y 725-733; Braun, 1979, pp. 105-117; Habermehl, 2004, pp. 7-108 y 178-188; Lazzati, 1956, p. 177; Lombardi, 1984, p. 27; Manaresi, 1914, p. 265, nota 1; Mazzucco, 1975, pp. 542-565; Musurillo, 1972, pp. 108-126; Puente, 1978, pp. 93-115; Siniscalco, 1983, pp. 103-104).

La defensa que con el testimonio y la ofrenda de su vida realizan los mártires cristianos, de la libertad personal y de conciencia a la hora de vivir y proclamar públicamente su fe es, a su vez, una ferviente profesión práctica de racionalidad: el mártir no padece el suplicio porque sí, o por ciega obediencia a un irracional mandato divino, sino que sabe, conoce y asume la razón de su decisión. Ni las argucias procesales ni el recurso a la quebradiza afectividad ni la invitación a la

3 "Un approcio solitarista non solo nega agli individui il diritto di scegliersi la propria identità, ma può anche essere un ottimo modo di non capire praticamente nessuno al mondo"(Sen, 2006, pp. 28-29). 
vacua y descomprometida simulación para cubrir la apariencia de una engañosa observancia de las disposiciones imperiales, consiguen quebrar la autenticidad de la consciente opción por la libertad del mártir cristiano (Calvo, 1994, pp. 99-100).

\section{Racionalidad y razonabilidad del martirio}

Esta dimensión racional y consciente de la fe cristiana es tan importante y fundamental para las primeras comunidades cristianas que el propio Tertuliano cierra su emblemático Apologeticum con dos bellísimos capítulos dedicados a demostrar el sentido y la lógica de la actitud martirial cristiana, a la vez que señala cómo la irracionalidad y la ausencia de sentido lógico son, por el contrario, características evidentes de quienes persiguen y asesinan a los cristianos. Sin embargo, dada la importancia de esta obra al momento de constatar y contrastar los problemas que padecieron, y las soluciones que aportaron las primeras comunidades cristianas, ante la necesidad de conformar su identidad en el ámbito de su pertenencia a la sociedad romana del Imperio, conviene recordar que tanto la biografía como la fijación cronológica de la obra de Tertuliano continúan siendo objeto de estudio y discusión entre historiadores y patrólogos que, trabajosa y concienzudamente, van poco a poco deshaciendo equívocos sobre el apologista y arrojando luz sobre su persona y personalidad (Braun, 1972, p. 70; Monceauz, 1901, p. 181).

El carácter apologético de su obra le obligaba, según la técnica griega de la apología, a asociar a todo tema apologético uno de carácter doctrinal, de exposición razonada de la fe, $\varepsilon \pi 1 \delta \varepsilon \imath \xi ı$ (Pellegrino, 1947, pp.1-65). Sin embargo, Tertuliano no limita su exposición a esta metodología escolásticamente dual o bipolar, sino que introduce un tercer elemento de crítica comparativa ( $\sigma \nu \gamma \kappa \rho ı \sigma \iota)$ entre la doctrina cristiana y sus contemporáneas no cristianas. Si bien Tertuliano utiliza géneros apologéticos preexistentes, la novedad metodológica de su obra reside en el esfuerzo por integrarlos sistemáticamente en una especie de sinfonía contrapuntística en la que, a partir de una dialéctica positiva y complementaria, la insistencia alternativa en cada uno de ellos no supone ni implica el olvido o la eliminación de los otros. Método que a su vez se presenta como prueba inequívoca de la importancia que Tertuliano atribuye a la razón y a la racionalidad a la hora de demostrar y justificar los contenidos de la fe cristiana; explica la propia estructura de la obra (Tertuliano, 1613, pp. 5-6; Paribeni, 1913, p. 37; Peterson, 1946, p. 355; Sordi, 1957, p. 89).

Tertuliano, al escribir su Apologeticum, busca, sobre todo, realizar una exposición detallada y de conjunto de la fe cristiana en la perspectiva metodológica y 
personal desde la que el autor planteaba el cristianismo como fides, disciplina y spes (Braun, 1964, p. 118). Al parecer, Tertuliano consideraba las dos primeras como comprensivas del Cristianismo, abarcando la primera el conjunto de las verdades de fe y comprendiendo la segunda todo lo relacionado con la vida moral y sacramental del cristiano en la Iglesia. Así pues, este carácter abierto y plural de la obra de Tertuliano constituye un elemento fundamental a la hora de entender e interpretar el significado atribuido por el escritor africano a conceptos o realidades básicas para la fe y la doctrina cristianas (Braun, 1964, p. 121).

En el capítulo 49 de su Apologeticum, Tertuliano pone de relieve la incoherencia de quienes califican la fe cristiana de supersticiosa, vanidosa y delictiva, mientras alaban, jalean y festejan ideas o creencias semejantes si son profesadas y defendidas por los no cristianos. Mas, cuando se trata del Cristianismo se llega a condenar una fe que, según Tertuliano, aunque se fundase en mentiras y supersticiones, es absolutamente necesaria para la sociedad, por su radical exigencia en buscar continuamente el bien; y se persigue y mata a quienes la profesan, mientras se la califica de vana, falsa e inocua (Tertuliano, 1613, p. 59). Incoherencia que se convierte en inútil y cruel irracionalidad cuando, al margen de toda razón, con la persecución de los cristianos solo se busca satisfacer las pasiones de la multitud popular, mientras que esta inconsecuente actitud de las autoridades refuerza y resalta el señorío de libertad, conocimiento, convencimiento y consciencia con que actúan los mártires (Tertuliano, 1613, p. 59). Cabe recordar que la insistencia en la fuerza educativa del Cristianismo, junto con las referencias a la historicidad de Cristo, en cuanto encarnación del Logos divino, y la justificación del rechazo radical de los cristianos del culto al emperador, han llevado a un buen número de especialistas a buscar en los Acta Apollonii una de las probables fuentes del Apologeticum de Tertuliano (Monceaux, 1901, p. 221-469; Musurillo, 1972, passim; Sordi, 1964, p. 169; 2004, p. 28; Traquandi, 1973, p. 133).

Pero es, sobre todo, en el colofón del capítulo 50 donde Tertuliano plantea directamente la razón, racionalidad y razonabilidad del martirio cristiano frente a la urdida y capciosa ironía de quienes defienden la inconsciente ceguera de la entrega cristiana de la vida o el oprobioso infundio de que al cristiano le agrada sufrir:

¿Por qué, pues, os lamentáis cuando os perseguimos, nos decís, si os gusta sufrir y debierais tener en gran estima a quien os facilita el sufrimiento, como deseáis? Es verdad. Aceptamos el sufrimiento: pero del mismo modo que aceptamos la guerra, 
que nadie acepta con gusto, por la angustia y desazón que produce. Y sin embargo, cuando ocurre, se lucha hasta el extremo de las fuerzas y quien al principio deploraba el combate, se alegra a su término de haber alcanzado la victoria, porque en ella ha conseguido gloria y botín. Nuestra guerra consiste en ser llevados ante los tribunales, para combatir a favor de la verdad, aun a riesgo de nuestra propia vida. Y nuestra victoria consiste en conseguir aquello por lo que combatimos: la gloria de agradar a Dios y la posesión de la vida eterna. ¡Pero nos derrotan! Ciertamente, pero después de haber vencido. Pues vencemos cuando nos matan y conquistamos la libertad cuando sucumbimos. $\mathrm{Y}$ aunque nos llaméis sarmentarios y nos consideréis carne de patíbulo, porque nos matáis en cruces de madera y nos quemáis en piras de sarmientos, así es como vencemos: ¡esta es nuestra túnica victoriosa, este nuestro carro triunfal! Es, pues, lógico que no agrademos a los vencedores; se entiende que nos tachen de locos y desesperados. $\mathrm{Y}$, sin embargo, esta misma desesperación, esta locura se transforma en símbolo de virtud y valentía cuando se utilizan para conseguir gloria y fama (Tertuliano, 1613, p. 60).

¿Por qué, se pregunta Tertuliano, no se reconoce a la actitud de los cristianos el mismo trato y consideración que la historia de Grecia y Roma atribuye a personajes que sacrificaron su vida en defensa de importantes valores humanos? ¿Por qué se reconoce la grandeza de sufrir por la patria, la propia nación, el imperio o la amistad y se ridiculiza y condena padecer por Dios mismo? ¿Por qué consideran insensato a quien de Dios espera la verdadera resurrección los mismos que intentan asegurar la resurrección y pervivencia a sus muertos por medio de estatuas, monumentos, epitafios e inscripciones? (Tertuliano, 1613, pp. 59-60).

No es la cómoda ceguera o el absurdo e ignorante conformismo lo que induce al martirio: la tolerancia y aceptación del dolor e incluso de la muerte, recuerda Tertuliano, es virtud reconocida y ensalzada por los propios filósofos y escritores considerados maestros por los propios perseguidores de los cristianos. Por ello, la contradictoria iniquidad del perseguidor se erige en el mejor testigo de la inocencia de los cristianos, precisamente por la obstinada cerrazón de las autoridades ante la consecuente actitud de los discípulos de Jesús que podría ser considerada como el triunfo de un convencimiento, de una idea trascendente que a la fuerza y al poder de los perseguidores únicamente opone la sangre de sus mártires; se trata, en la actitud martirial, de la victoria de una ley, la del amor en Cristo, que une a los hombres con y en un solo vínculo, a la vez que refuerza su capacidad de sufrir, transformando 
radicalmente, mediante la fe y la esperanza radicadas en la salvación de Cristo, el desgraciado, fatídico y sinsentido sufrimiento pre-cristiano por el convencimiento del triunfo definitivo de la libertad personal en la resurrección del alma y del cuerpo (Tertuliano, 1613, pp. 60-61).

\section{Las pertenencias racionales y libres}

En el ámbito de las pertenencias, sean sociales, religiosas o ideológicas, la racionalidad se erige en clave y fundamento que diferencia la víctima (arracionalidad, ausencia de libertad y padecimiento) del mártir (consciencia, libertad y voluntariedad): solo la aceptación consciente y racional/razonada de la muerte funda, fundamenta y legitima el martirio. Libertad (identidad) y pertenencia que también inspiraron la actitud de los cristianos con relación a las autoridades imperiales al no reconocer al César su pretensión de autoridad totalizadora, y no solo última o suprema, sobre sus súbditos, como se deduce de los textos de S. Clemente Romano, Carta a los Corintios, 60, 4 y 61, 1-3; San Justino Mártir, Apología, I, 17; Teófilo de Antioquía, Ad Autolicum, I, 11 (Rahner, 1990, pp. 46-47). En razón de esta tendencia totalizadora los Emperadores unían y centraban en su persona, política y religión, Estado y divinidad. Al autoproclamarse gens divina no secularizaban la divinidad sino que, por el contrario, divinizaban cualquier posible resquicio de secularidad social y política. Sin embargo, y de ahí la posibilidad de la existencia de pluralidad de pertenencias a partir de una identidad fundamental prioritaria, desde un principio los cristianos aceptaron sin ningún tipo de reparo o reticencia, en todo lo referido a la convivencia civil, las estructuras jurídico-políticas del Imperio y siempre procuraron evitar exquisitamente cualquier atisbo de colisión, oposición o disconformidad con las mismas (Peretto, 1989, pp. 89-114). Tertuliano es quizás el primer gran representante de quienes buscaron desde el principio cauces para un auténtico entendimiento de convivencia entre la identidad cristiana y la pertenencia simultánea, junto a su fe, a la civitas romana (Cochrane, 1940, pp. 324-415; Frend, 1965, pp. 98-129; Guignebert, 1901, passim; Hornus, 1960, pp. 112-174; Klein, 1968, passim; Perretto, 1989, pp. 89-114; Powell, 1975, pp. 33-54; Rordorf, 1969, pp. 105-141).

Esta, que podríamos denominar razón de totalidad en las autoridades imperiales les condujo, por ejemplo, en la planificación de las persecuciones, a intentar, sobre todo, hacer apóstatas y no mártires, entre los cristianos, y de este modo, conseguir, 
en el hecho de la apostasía, una prueba incontestable de aceptación del poder totalizador del Emperador (Calvo, 1994, p. 103). Consciente o inconscientemente, las autoridades imperiales habían percibido que el enfrentamiento entre cristianos e imperio trascendía lo anecdótico o cotidiano para fondear en el irreductible contraste de dos concepciones totalizadoras del mundo jurídico y de la vida social y política (Andonegui, 1993, pp. 433-467; Lombardi, 1986, p. 4-5, nota 5), si bien las perspectivas desde las que una y otra se articularon no fueron ni homogéneas ni homologables ni reducibles una a otra (Lombardi, 1984, p. 92).

En la medida en que la pertenencia y la identidad eran dos realidades necesaria y uniformemente relacionadas para las autoridades romanas, el cristianismo y el imperio estaban abocados a enfrentarse. Y no porque fuesen, en principio, radicalmente contradictorios, sino exclusivamente porque el absolutismo de los Césares, que pretendía abarcar todos los aspectos y dimensiones fundamentales del ser humano, excluía, por su propia esencia, cualquier atisbo público de libre autonomía personal, requisito totalmente esencial y requerido en la fidelidad al seguimiento de Jesucristo, única razón y sentido de la comunidad de creyentes (Gigon, 1970, pp. 253-254). La radicalidad de la pertenencia cristiana y la libertad de la identidad del cristiano que le abre a la aceptación de, así como a su integración en, las más variadas estructuras sociales, estriba, sobre todo, en el hecho de que la adscripción a la comunidad cristiana no se basa en ideología alguna, sino en la pertenencia sacramental. Desde los primeros tiempos del cristianismo fue una realidad indiscutida que el Bautismo, sacramento que posibilita la recepción de todos los demás, es a su vez, el que hace real y efectivamente la adscripción del fiel a la comunidad eclesial y en consecuencia, el que produce y genera esencial, imprescindible, fundamental y absolutamente la pertenencia a la comunidad eclesial, al margen y por encima de ideas, creencias u otras pertenencias menores (Calvo, 1990, pp. 117-118; Di Berardino, 2002, p. 123; Bianchini, 1986, pp. 241-263; Staats, 1972, pp. 29-52; 1975, pp. 242-263).

Fue tan importante para los cristianos, al menos en los primeros siglos del Cristianismo y sobre todo a partir del siglo IV, la búsqueda y el mantenimiento de su identidad, dentro del ámbito y respeto de su propia pertenencia al Imperio, que el sentido de pertenencia/identidad cristiana provocó y culminó el proceso secularizador de los juegos circenses y su total desvinculación de cualquier referencia o significación religiosa, como lo atestiguan las leyes que entre los años 342 y 469 regularon la protección del calendario festivo cristiano, haciendo desaparecer toda referencia, por 
mínima e indirecta que fuese, a festividades paganas dentro del calendario/programa de los juegos circenses, separando radicalmente el juego de cualquier connotación sacra o religiosa. E1 Cristianismo seculariza los juegos, curiosamente, porque los "despaganiza”, aislándolos e independizándolos de toda relación con o motivación en fiestas religiosas no cristianas (Jiménez, 2003, p. 25; Saggioro, 1999, pp. 103-131; 2003a, p. 165; 2003b, pp. 65-80; Zerbini, 2003, pp. 81-90).

No puede faltar una breve referencia al Codex Theodosianus (Código Teodosiano), primera codificación jurídica oficial y auténtica, que de forma indirecta, ofrece en primicia, desde y en el ámbito del derecho vigente, un conjunto de elementos esenciales a la hora de precisar el propio concepto de identidad cristiana (Archi, 1976, pp. 52-67). Esta importante compilación de normas respondió de un modo directo a la situación provocada por el ya, a principios del siglo $\mathrm{V}$, exagerado e incontrolado aumento de leyes con la negativa consecuencia de su compleja y contradictoria divergencia; como lo expresaba con meridiana claridad el autor anónimo del De rebus bellicis en pleno siglo IV:

Divina providentia, sacratissime Imperator, domi forique rei publicae praesides comparatis, restat unum de tua serenitate remedium ad civilium curarum medicinam, ut confusas legum contrariasque sententias, improbitatis reiectio litigio, iudicio augustae dignationis illumines. Quid enim sic ab honestate consistit alienum quam ibídem studia exerceri certandi ubi, iustitia profitente discernantur merita singulorum? (De rebus bellicis, XXI y Giardina, 1989, pp. 97-127).

El ordenamiento jurídico no podía permanecer ajeno a las vicisitudes históricas de una época en las que valores, ideas y actitudes se habían transformado radicalmente. Cambio y novedad que, institucionalmente, se plasmaba en la transmutación del imperio romano-pagano en un imperio romano-cristiano (Leg. III, 1-3 y Nocera, 1983, pp. 28-35). En este contexto socio-político, el Codex Theodosianus constituye el primer intento por parte de la autoridad imperial de realizar, con todas sus consecuencias, un proyecto legislativo unitario y universal en el ámbito del Imperio (Nov. Theod. I, 1: De Theodosiani codicis auctoritate y Volterra, 1984, p. 3086), a partir de un novedoso principio de unidad que, en la práctica, consiguiese la síntesis "entre las exigencias legislativas estatales que configuraban la identidad de la vieja estructura social, tradicional e intrínsecamente pagana, con los principios de identidad de la cultura cristiana" (Saggioro, 2003a, p. 171). 
La evolución que configura y explica la identidad cristiana, desde la perspectiva del derecho imperial y la propia autocomprensión del Cristianismo, está indisolublemente relacionada con su conformación y crecimiento como y en cuanto comunidad, es decir, un grupo radicalmente unido en su dimensión y esencia religiosa, en el sentido de que participan de un conjunto de valores y creencias comunes que, a su vez integran y se integran en un cuerpo doctrinal orgánicamente sistematizado y racionalmente especulativo en torno al ser y sentido del hombre y del mundo. En este orden de ideas y aunque en el Código Teodosiano jamás se confunden Imperio y Cristianismo, dicha codificación, por su alineación funcional con el Cristianismo, establece una definición legal, en el sentido del derecho romano, de la religión cristiana; avalada por el Código que aportó elementos y medios fundamentales a la hora de configurar y delimitar la identidad cristiana; por ejemplo, el hecho de la inversión socio-política que se opera a partir de la entrada en vigor del documento, porque en ese momento, comienza a existir conceptual y terminológicamente el paganismo, paganitas, en cuanto religión alternativa al Cristianismo, christianitas (Sabbatucci, 1988, pp. 42-45). Así pues, al ser considerado el Cristianismo fides católica, y por tanto Religión de Estado y del Estado, la fe cristiana asume el papel de único principio religioso posible en cuanto fundamento de la dimensión religiosa del pueblo ( $C$ Th XVI, 11). De este modo, como hemos apuntado, en el caso de los juegos, determinados valores y comportamientos que formaban parte de la realidad cultual romana, aunque conservados, se sitúan fuera y al margen de la realidad religiosa, en razón de su absoluta falta de correspondencia con la religión cristiana basada en y promotora de valores y principios completamente diferentes.

\section{Relación identidad-pertenencia y diversidad socio-cultural}

Ante la posible pregunta por la presencia de la religión, de la religiosidad y de todo lo que implican y connotan, de forma especial, en las sociedades avanzadas de nuestro siglo, hemos de convenir en primer lugar en que los pronunciamientos realizados en las últimas décadas sobre la desaparición o privatización de lo relacionado con la religión, se han diluido en el espejismo de la ilusión secularizadora ignara de su quebradiza ambivalencia (Baubérot, 2001, pp. 634-635). Y con cierta sorpresa no exenta de admiración, experimentamos que en estas sociedades pluriculturales (Calvo, 2003b, pp. 161-217) la religión vuelve a ocupar un lugar relevante en la esfera pública. Antes de abordar nuestra reflexión sobre la incidencia de esta novedad 
pluricultural y pluriconfesional en la conformación de pertenencias e identidades, ofreceremos algunos matices sobre conceptos de uso común entre sociólogos y juristas que por su radical polisemia, hacen muy difícil, incluso imposible a veces, la solución o superación de determinados problemas o contradicciones. Me refiero, sobre todo, a los tan recurridos conceptos de multiculturalismo y pluralismo (Calvo, 2003c, pp. 73-106).

a. La diversidad cultural y religiosa elemento configurador de la pertenencia social

Como sucede siempre que nos encontramos ante un neologismo creado para denominar o describir algo no formulado y contrastado en su totalidad, mediante el término multiculturalismo suele designarse una realidad de complicada definición (Sartori, 2001, pp. 55-73 y 25-45). Significa, por una parte, el hecho de reconocer igual dignidad a las expresiones culturales de todos los grupos y comunidades que conviven en una sociedad democrática, abarcando también, desde una dinámica más operativa, el significado, las justificaciones y las consecuencias de tal reconocimiento. Pero, del mismo modo se aplica a la idea de que todo ser humano tiene derecho a crecer y desarrollarse dentro de su propia cultura y no en aquella en que intenta asimilarla una mayoría social ajena a sus pertenencias culturales, sociales o religiosas.

El multiculturalismo, considerado como mera verificación fáctica de la existencia de diferencias culturales, ideológicas o religiosas en el ámbito de una sociedad, no tiene por qué plantear problema alguno, en la medida en que se trataría simplemente de la descripción de un hecho; o dicho de otro modo, de la simple constatación de una sociedad plural. Las dificultades surgen cuando en la explicación o justificación de una sociedad formada por culturas distintas se acude al multiculturalismo como y en cuanto valor, pues, a partir de este momento, se convierte en ideal social, con la consiguiente revisión y transmutación de valores y fines sociales, lo que realmente no era más que un hecho o coyuntura social (Llamazares, 2002, pp. 176-177).

Precisamente por hallarnos ante una realidad no contrastada social, filosófica y jurídicamente, son abundantes los problemas e interrogantes con que se enfrenta cualquier intento de reflexión sobre las cuestiones relacionadas con o derivadas del multiculturalismo: de qué tipo de reconocimiento social y jurídico se trata cuando nos referimos a la igualdad cultural; qué quiere decir o qué se pretende significar cuando se habla de igual dignidad; cómo se han de concebir e integrar, en caso 
de aceptarla, la existencia de derechos colectivos en el marco de las constituciones democráticas vigentes; a qué tipo de grupos o comunidades le han de ser atribuidos y reconocidos determinados derechos colectivos (Sartori, 2001, pp. 25-27 y 65-72). Desde el ámbito de las ciencias positivas y de la naturaleza, y del mundo de lo social, en el siglo XX se ha planteado la gran dificultad, imposibilidad en tantos casos, que entraña el descubrir fundamentos de carácter universal y de validez general. Cada vez se hace más conflictivo plantear la validez de un deber ser vinculante para todos y al mismo tiempo, parece ser una constante que el avance social e industrial de las sociedades se desvanezca, progresivamente, la creencia de que la propia ética y los valores supuestos e implicados puedan ser propuestos como una moral universal (Macintyre, 1995, pp. 209-228).

Probablemente, la idea-madre que subyace a la elaboración de este concepto radique en el convencimiento de que para el Estado no solo existen ciudadanos, sino personas, en la medida y sentido en que se acepte que determinadas dimensiones o características sociales, religiosas, etnológicas o culturales integran la realidad personal, a pesar de que implique asumir la confusión e identificación entre persona y personalidad. Esto equivale a decir que las instituciones públicas deben valorar y tener en cuenta todo, por accidental que fuera, lo que diferencia a un individuo de otro. De este modo, el Estado contemporáneo se ve forzado a contrastar su legitimidad/racionalidad no solo con aquello que se refiere a la protección de los individuos y sus derechos subjetivos, sino también en lo que afecta a los elementos estructurales e ideológicos de aquellos grupos o comunidades que reivindican y reclaman su reconocimiento y aceptación socio-jurídicas. Se opera, así, una inversión institucionalista o corporativista en el que la identidad colectiva sobrepasa, hasta acabar imponiéndose en algunos casos, al individuo: las relaciones Estado [derecho] - individuo inician en todos sus ámbitos y especificidades, un proceso de institucionalización-despersonalización, primando los hipotéticos derechos de una ficción jurídica, la cultura, sobre los de una realidad jurídica: la persona-individuo.

Esta opción multiculturalista por el ideal del respeto a la identidad de todas y cada una de las comunidades que conforman una sociedad, transfiriendo y transmutando las propiedades y exigencias de las identidades personales en identidad social, hace mucho más difícil el funcionamiento y efectividad de leyes e instituciones que deben valer y ser iguales para todos, más allá de limitaciones o confines colectivos o comunitarios. Las causas sociales o económicas han pasado a un segundo plano a la hora de determinar o delimitar la estratificación social, 
mientras que en sociedades consideradas más avanzadas por su economía o por su situación geopolítica, ocupan el primer plano en el orden de la identidad/diversidad, pertenencia/relación, motivos y características de orden cultural, hasta el extremo que cada vez con más virulencia nuestras sociedades se ven convulsionadas por problemas o movimientos de carácter identitario, y por ende, segregacionistas o culturalmente "racistas", ya se funde la identidad en cualidades o circunstancias sociales, culturales o religiosas o bien en infundadas pretensiones democráticas (Curtis, 2006, pp. 15-66 y 95-185; Lee, 1996, pp. 36-107; Lincoln, 1994, pp. 3262; Pace, 2003, p. 117). Los teóricos del multiculturalismo, sin embargo, van más allá al hablar no solo de igual dignidad entre culturas, atribuyendo a una entidad no personal (cultura) una propiedad o cualidad especificadora y determinante de la persona, sino que, en lógica consecuencia, al aceptar la dignidad de las culturas se aboca inevitablemente al problema de los derechos culturales, ya sean entendidos contra, extra o secundum personam (Gutmann, 1992, passim; Kymlicka, 1992, pp. 140-146; 1995, pp. 10-48 y 108-172; Margalit y Halbertal, 1994, pp. 491-510).

Kymlicka distingue tres formas, versiones o clases de derechos culturales (Kymlicka, 1995, pp. 10-48). La exigencia del reconocimiento de los derechos culturales equivale, por ejemplo, a reclamar el derecho de una minoría étnicocultural al gobierno, a través de la autonomía política, en un territorio en que aquella resulte socialmente mayoritaria, que les permita, en cuanto grupo, adoptar todas las medidas para desarrollar óptimamente la propia cultura. Otras veces, los derechos culturales aparecen bajo la exigencia de protección jurídica de la libertad de expresión de elementos típicos y constitutivos de la identidad de una minoría cultural, como ocurre, por ejemplo, en la reivindicación de ciertas prendas de vestir (el turbante de los Sikh, el chador, etc.), del respeto de los valores de las minorías en los currículos escolásticos y en los medios de comunicación, de la financiación pública de actividades características e identificadoras de la comunidad étnica (Lamnbin, 1999, passim). También los derechos culturales pueden adquirir la forma de derecho a una representación especial en el seno de instituciones legislativas, administrativas o educativas, a través de un sistema de cuotas, con el consiguiente riesgo de perversión despersonalizadora, a través de la hipertrofia de intereses grupales o institucionales, de los medios democráticos de representación política, mediante el predominio o prevalencia de la pertenencia sobre la libre configuración de la identidad personal. Existen, además, al menos otros dos tipos de derechos culturales: los que se articulan como medidas de autoprotección interna, intragrupal 
o intracomunitaria; y los que definen y protegen al grupo en relación con otros grupos o comunidades. Se encuadran en el primer apartado las normas que autorizan al grupo étnico o a los miembros de una determinada cultura a controlar, con o sin el auxilio de lo público, a los propios correligionarios o miembros del mismo grupo. Pertenecen a la segunda acepción las normas (a través del autogobierno, las cuotas o la representación especial) dirigidas a evitar que centros de decisión, institucionales o no, ajenos al grupo o comunidad acaben determinando sus destinos de forma incontrolada (Ferrara, 1992, passim), incluso a riesgo de que política y socialmente lo orgánico-institucional (pertenencia) acabe prevaleciendo sobre lo personal (identidad).

A excepción de los argumentos que explican la existencia de los derechos culturales a partir del reconocimiento y la consolidación de la identidad de grupos y personas, el peso argumentativo de los razonamientos con que se justifica su defensa y promoción radica, sobre todo, en la libertad y la igualdad en cuanto valores del Estado de derecho. Cada vez con más fuerza y razón, la justificación histórica de los derechos culturales, basada en el respeto a acuerdos pactados entre naciones que viven en el mismo Estado, así como la que los defiende por el hecho de considerar el pluralismo y la diversidad como bienes en sí mismos, se estiman más como argumentos menores o secundarios que legitiman; pero de ningún modo pueden ser entendidos como su fundamento y razón la existencia de estos derechos (Kymlicka, 1992, pp. 140-146; Kymlicka, 1995, pp. 15-60 y 108-152). La protección de la cultura, pues, se justificaría desde el horizonte interno de los propios miembros pertenecientes o integrados en ella: realmente, al proteger una cultura, se estaría ofreciendo cobertura a su capacidad para dar sentido y significado a la vida de los deudores de la misma (identidad) por estar en ella inmersos; o lo que es lo mismo, para posibilitar el desarrollo óptimo de la identidad personal de quienes a ella pertenecen.

Al privilegiar o potenciar de este modo la dimensión individual-personal (identidad) como motivo o fundamento del reconocimiento jurídico de las particularidades sociales de un grupo determinado, puede parecer que se quiebra el principio de igualdad. Sin embargo, recuerda Kymlicka que el reconocimiento de estos derechos y prerrogativas culturales es necesario en razón y en la medida en que la realización efectiva de la justicia exige y requiere que se renueven y reequilibren las discriminaciones moralmente arbitrarias, especialmente si son profundas, perversas y por razón de nacimiento; pues si no existiesen estos derechos especiales, 
los miembros de las minorías culturales no dispondrían de las mismas posibilidades de vivir y trabajar" en las mismas condiciones personales y sociales en que lo hacen "quienes pertenecen a culturas mayoritarias (Kymlicka, 1992, p. 146). Desde esta perspectiva, el multiculturalismo puede ser defendido a partir del valor de la igualdad, integrador de la identidad en la pertenencia, en cuanto reconocimiento explícito, dentro de un sistema político, de idéntica dignidad y protagonismo a realidades culturales y étnicas distintas, sin que políticamente se prime a ninguna cultura sobre otra (Waldron, 1992, pp. 751-793).

A pesar de su aparente éxito, sobre todo si nos atenemos a su continua invocación por parte de políticos y medios de comunicación, hemos de hacer referencia a los posibles riesgos que subyacen a esta especie de funcional deus ex machina social en que se ha convertido el multiculturalismo. G. Kateb, en un profundo y cuidado análisis de los riesgos inherentes a la institucionalización de una sensibilidad multiculturalista, ha enumerado y estudiado una serie de vicios o quiebras en la pertenencia social que, de un modo u otro, reconocen en el multiculturalismo su origen y desarrollo (Kateb, 1994, pp. 525-530). Pero, desde la perspectiva de esta reflexión, nos interesa más la crítica realizada por $\mathrm{Ch}$. Kukathas, sobre todo cuando después de advertir que la protección jurídica de la especificidad cultural puede ser utilizada, ex post ipso, como autolegitimación alienadora por parte de grupos constituidos meramente sobre la base de un interés excluyente de cualquier otro grupo o persona, señala el riesgo que corre el multiculturalismo de fijar, de congelar, al grupo protegido en su contingente configuración actual, impidiendo cualquier proceso de revisión interna de su cultura puesto que

los grupos culturales no son conjuntos indiferenciados, sino asociaciones de individuos con intereses divergentes. Dentro de estos grupos minoritarios existen otras minorías más pequeñas. Considerar al grupo en su conjunto como sujetos de derechos culturales es lo mismo que aceptar como definitivas las estructuras existentes e indirectamente, favorecer las minorías-mayoritarias existentes y tal como existen (Kukathas, 1992, p. 114; Maffettone, 2006, pp. 64-67).

De este modo, se asumiría el riesgo, evidente y asfixiante en tantas situaciones, de predominio socio-político de nacionalismos excluyentes, de convertir en sociedades cerradas grupos socio-culturales que se habrían garantizado su supervivencia por vivir en una sociedad abierta. Nos hallaríamos ante un tipo ideal y modélico del 
movimiento dialéctico que mientras se reafirma la cohesión-identidad del grupo (pertenencia), el derecho a la diferencia, a su promoción y respeto, se conforma la vigencia e influencia de un principio disgregador del moderno Estado de derecho, al producirse una irremediable quiebra de la igualdad (Pace, 2003, p. 115). $\mathrm{Cu}^{-}$ riosamente, entre los remedios que ofrecen los promotores del multiculturalismo para evitar estos riesgos, solo se señala uno como más radical y efectivo, cargado, a su vez, de un inoperante voluntarismo: el derecho de abandono, garantizado al individuo siempre y en cualquier circunstancia. Ahora bien, esta opción conduce necesariamente a subordinar cualquier medida protectora de una cultura al hecho de que la comunidad o el grupo reconociese a sus miembros la libertad de abandonarlo sin sufrir represalias. Principio ineficaz e inefectivo en aquellos casos y sociedades para los que sería más urgente y necesaria su invocación (Holmes, 1994, pp. 599610; Pace y Guolo, 2002, pp. 3-10 y 29-61; Paolucci y Eid, 2006, passim; Sennett, 1977, pp. 28-63). Ello supone, en la práctica, dejar abierta una de las vías más importantes para la permeabilización entre culturas distintas que es, precisamente, una de las más importantes características del pluralismo.

\section{b. Pluralismo, identidad personal y pertenencia social}

Se suele entender por pluralismo el conjunto de circunstancias sociales y actitudes personales que conforman y delimitan ámbitos de convivencia en los que el poder político, económico, cultural, ideológico, etc., está equitativamente distribuido, compartido, reconocido, contrastado y controlado. En la medidaen que una sociedad es plural, se torna innecesaria la tolerancia, como actitud, como hecho; activa (ser tolerante) y pasiva (ser tolerado); vertical (tolerancia hacia el inferior); u horizontal (tolerancia respecto al igual), porque cada cual se mueve libremente en el ejercicio de sus capacidades, la expresión de sus opiniones, la puesta en práctica de sus ideas y la profesión activa de sus creencias a través de determinados comportamientos; es decir, estamos frente a lo que se denomina, en perífrasis más profunda y comprometida de lo que pudiera parecer a primera vista, democracia como forma de vida o como cultura. Es decir, se trata de la democracia "no solo como un específico régimen de organización y gobierno político, sino ante todo como una cultura global y universal, como forma de vida individual y comunitariamente valiosa" (Querejazu, 1994, pp. 48-49; Calvo, 2003a, pp. 93-114).

No es fácil, por interesado que sea el esfuerzo, definir o delimitar en y desde todas sus perspectivas e implicaciones al pluralismo. Dentro de la compleja red de 
relaciones en que se articula este concepto, nos interesa señalar aquí, sobre todo en cuanto perspectiva metodológica, su dialecticidad, es decir, el hecho de que los requisitos, condiciones y presupuestos que posibilitan el ejercicio del pluralismo se profundizan, maduran y enriquecen, sobre todo, con su ejercicio y puesta en práctica. Entendido así, el pluralismo enraíza y se consolida en una concepción del hombre basada, desde el convencimiento jurídico-formal y el reconocimiento práctico-material, en la dignidad humana, así como en los atributos y características en ella implicados y en los derechos personales y sociales que de ella derivan. De este modo, el pluralismo únicamente es posible en condiciones, formales y materiales, de igualdad y libertad (Querejazu, 1994, pp. 50-54), como única posibilidad de realización material y formal de la justicia. Preguntarse por la naturaleza, el significado o los contenidos del pluralismo equivale a acercarse a una cuestión que afecta a todas las dimensiones del hombre y de la sociedad.

Cada persona es "identitariamente" un todo único y singular. Unidad y unicidad que, hemos de reconocer, son la fuente y origen del profundo deseo que cada ser humano tiene de ser y verse reconocido en su dignidad y singularidad o lo que es lo mismo, en su identidad. Es una constante en la experiencia personal que precisamente, el reconocimiento social (pertenencia) es la mejor garantía de estima personal (identidad). Sin embargo, no puede olvidarse la paradoja en que se desenvuelve la naturaleza humana al necesitar perentoriamente de otras realidades y de otras personas, es decir de la sociedad, para alcanzar y satisfacer sus fines personales. Esta dimensión social de la naturaleza humana se traduce y concreta en la existencia de una verdadera y auténtica comunidad humana; y si se puede hablar de comunidad es porque en cada uno de sus miembros existe, más allá o además de sus exclusivas características o propiedades personales, un constitutivo común con los demás.

La progresiva importancia que en el mundo actual se atribuye a los derechos del hombre es muestra incontestable de cómo lo que existe de común en las personas prevalece sobre lo singular y particular, dado que la alteridad no es solo ni principal característica o propiedad de la naturaleza humana, sino constitutivo esencial de su ser-persona: miembros de una misma comunidad, los aspectos particulares y plurales o disgregadores (raza, cultura, ideología, religión, etc.) no pueden eclipsar el fundamento que nos relaciona esencial y radicalmente a unos con otros. Para que una sociedad pueda considerarse pluralista ha de estar integrada en sus miembros por personas que hayan accedido libre y voluntariamente, sin que su ingreso o salida se impida por ningún motivo de exclusividad: apertura y libertad son las 
dos condiciones que caracterizan a una sociedad como pluralista, que no significa mera equivalencia o equipolencia con la variedad o pluralidad de sociedades. E1 pluralismo social no es cuestión numérica, cuantitativa o de simple variedad, sino de carácter cualitativo, interno, de estructura, constitución y funcionamiento de cada sociedad concreta.

Desde una perspectiva más amplia de las ciencias sociales, se ha de tener en cuenta que en la ciencia política y en la sociología, se ha entendido por pluralismo, la doctrina política opuesta a la teoría monista del Estado, o una hermenéutica de análisis y comprensión de las sociedades e instituciones humanas distintas del Estado. El pluralismo moderno, tal y como se ha desarrollado en Inglaterra y América entre los años 1900 y 1930, surgió como consecuencia de la imposibilidad estructural que atenazó al Estado moderno a la hora de dar respuesta a importantes problemas surgidos de modos de organización social hasta entonces desconocidos, junto a las nuevas formas de articulación institucional de los Estados, tanto ad intra como ad extra. La teoría del Estado soberano, elaborada principalmente por juristas, provocaba graves dificultades desde el punto de vista político, sobre todo, en la medida en que soberanía se confundía con omnipotencia del Estado. El desacreditado Estado soberano fue el principal objetivo crítico de las teorías y doctrinas pluralistas que, desplazando su interés y punto de mira del Estado a la sociedad y grupos sociales, reivindicaron derechos y protagonismo social para grupos profesionales y asociaciones, en oposición al Estado (Duguit, 1911, passim). Tampoco ha sido ajeno a esta consolidación de la práctica democrática el progresivo reconocimiento de los derechos del hombre que, en cierto modo, ha despolitizado, en su sentido más estricto, el concepto de democracia para convertirlo en modo o marco para delinear o tipificar las relaciones humanas, asumiendo como fundamento, el respeto a la igual dignidad de todos los hombres y a sus ideas, aun cuando no se compartan: este precisamente sería el punto de inflexión en que se entrecruzan tolerancia y pluralismo.

\section{Conclusiones: de la racionalidad en la pertenencia a la identidad personal}

Si el ser humano solo puede desarrollarse en plenitud a partir de una continua referencia social, en la relación necesaria con el/los otro/s, la pertenencia, según hemos apuntado en estas páginas, se convierte en medio prioritario de conformación 
de la propia identidad. Directamente o a través de su papel determinante en la configuración de los conjuntos culturales con que se relaciona, la religión es uno de los elementos más influyentes en los ámbitos sociales que determinan y conforman las relaciones de pertenencia. La íntima y profunda relación que guardan pertenencia social e identidad personal dispone la fundamental incidencia que las relaciones sociales de pertenencia tienen en la formación y desarrollo de la personalidad, entendida, por lo que aquí respecta, en su especificidad de identidad personal. Por ello, cabe inferir que únicamente podrán ser consideradas como auténticamente personalizadores aquellos modelos de pertenencia en que se reconozca un papel esencial a la dimensión racional de la persona, que podríamos denominar como pertenencia crítica, siempre enriquecedora y promotora de la identidad personal, al favorecer, fomentar y promover la autocrítica, personal y colectiva, y la heterocrítica social e institucional; frente a un tipo de pertenencia más de carácter socio-afectivo, donde primarían las actitudes irracionales o arracionales.

La pertenencia religiosa, precisamente, constituye un excelente banco de pruebas para comprobar cómo y hasta qué punto la racionalidad o razonabilidad condiciona y establece la virtualidad personalizadora de la pertenencia social. Las relaciones sociales que el derecho mantiene y ha mantenido con las religiones, en el caso concreto de nuestras naciones y área cultural con el cristianismo, judaísmo e islamismo, pueden servirnos de indicio pedagógico para delimitar con mayor precisión en qué sentido hablamos de pertenencia crítica o racional y de pertenencia socio-afectiva o irracional. La sociedad en que nació y se difundió el Cristianismo, tanto en su vertiente oriental como occidental, era regida y ordenada por un sistema jurídico muy elaborado y totalmente marginal a la nueva fe, el derecho romano. Los predicadores del Evangelio jamás lo presentaron como un conjunto de normas, reglas o prescripciones con la pretensión de sustituir o anular ningún sistema jurídico o cuerpo legal vigente en la sociedad de su tiempo:

Mientras en Europa, a pesar del predominio de la Iglesia, el derecho representaba una fuerza relativamente autónoma (recordemos la doctrina de las "dos espadas", la de la Iglesia y la del poder secular, la rivalidad y la lucha entre el papado, que tenía pretensiones de dominio teocrático, y el poder estatal), en el mundo árabe el derecho constituía parte imprescindible de la religión; el derecho islámico no conoce diferencia entre derecho canónico y laico, todo derecho es aquí derecho de la comunidad de creyentes (...) Dado que los musulmanes no elaboraron un derecho 
puramente laico, precisamente en razón de su naturaleza sacra, el derecho no era capaz de adaptarse a los cambios sociales, constituyendo de este modo una de las más importantes fuerzas conservadoras. En los países musulmanes el desarrollo del derecho cesó ya en el siglo $\mathrm{X}$, es decir, cuando estaba comenzando el gran desarrollo del derecho medieval europeo (Gurevich, 1972, p. 164).

Situación que claramente contrasta con la doctrina mosaica o con la predicación de Mahoma, dirigidas a grupos humanos socialmente invertebrados donde el derecho como fenómeno social articulado era prácticamente inexistente. Por ello, el Pentateuco y el Corán no son considerados únicamente libros religiosos, sino también recopilaciones, colecciones de reglas de derecho y de normas puramente temporales, aunque de exclusivo origen divino, y reguladoras de aspectos estrictamente socio-temporales y políticos de la actividad personal.

La pertenencia evangélica, al orientarse primaria y directamente a una dimensión personal y comunitaria distinta, por principio, de aquella en que se desenvuelven las instituciones exclusiva e inmediatamente jurídicas, posibilita, facilita y urge la observación racional, sin atisbo de imposición extra o metarracional alguna, de sus diversas pertenencias, incluida la religiosa, pues la auténtica conversión al Evangelio se basa y es imperiosamente consecuencia de la libertad humana, es decir, de la más razonada actividad de la persona. Por su propia esencia, el cristianismo exige y se funda en pertenencias críticas, conscientes, racionales y personalizadas, como único medio válido de situar a la persona y su dignidad por encima de cualquier pertenencia social derivada y secundaria, en razón de su radical trascendencia, derivada de la inhabitación personal del Espíritu (Malaurie, 2003, po. 622-627).

En esta dimensión de racionalidad de la pertenencia, adquiere su sentido más pleno y especial la obligada referencia a la aceptación, observancia y promoción de los derechos humanos como criterio de pertenencia social crítica, en cuanto a que la convergencia sobre la validez y efectividad de los derechos humanos garantiza la racionalidad y la libertad en toda opción que se realice por una determinada pertenencia, dado que no siempre y en todas las personas y circunstancias la elección y las preferencias personales son coincidentes (Maffettone, 2006, pp. 79-80). La convergencia posible y necesaria en cuestión tan importante, cual la aceptación de la validez general de los derechos humanos, es prueba inequívoca de la importancia del principio de que toda identidad personal, como hemos mencionado, se conforma como identidad plural y a su vez, en la medida en que es aceptada y asumida, 
implica la libertad crítica para decidir sobre el valor e importancia que, según los contextos en que se hallen, puede atribuirse a las diferentes asociaciones, afiliaciones y opciones sociales, culturales y religiosas que enmarcan y condicionan las diversas y posibles pertenencias. La racionalidad crítica se actualiza y ejerce cuando existe la posibilidad de una pertenencia libre y consecuentemente responsable, resultado de una elección sin predeterminaciones o imposiciones; y al contrario, se convierte en irracional cuando las relaciones humanas, entre personas de distintas identidades, se reducen, aun en los supuestos en que sobre todo prevalece la buena fe, a términos de amistad o alianza de civilizaciones, o bien de diálogo entre los distintos grupos religiosos, e incluso de relaciones de amistad entre comunidades diversas en sus principios y modelos de comportamiento. Al ignorar la gran diversidad de formas y modos con que las personas se relacionan entre sí, el ser humano queda empequeñecido, desfigurado y reducido a un número ignoto dentro de un dudoso protagonismo del grupo o de la institución.

La irracionalidad acrítica en las pertenencias sociales suele manifestarse, sutil pero patológicamente, cuando ante las decisiones y actitudes que parcializan el mundo se intenta su unificación a través de su uniformidad a través de clasificaciones dominantes, exclusivas y excluyentes en términos religiosos, culturales, nacionales, de creencias o de civilización, considerando, según convenga, a cada uno de estos factores como el único determinante de los grandes conflictos que actualmente nos interrogan y urgen (Sen, 2006, p. 31; Pace y Guolo, 2002, pp. 110-128). La actitud de quienes, por ejemplo, recurren a la pertenencia religiosa como factor generador de determinados conflictos de índole social constituye muestra inequívoca de esta incongruente irracionalidad, pues al mismo tiempo que se hipertrofia la repercusión social del seguimiento religioso como causa de conflictos, se priva, por principio, a la religión de su dimensión pública reduciéndola al ámbito del más privado individualismo espiritualista y cercenando, además, como consecuencia de todo reduccionismo totalitario, una de las relaciones de pertenencia más fundamentales de la persona. Irracionalidad en la concepción y valoración de las pertenencias religiosas que en algunos casos ha adquirido formas y expresiones grotescas. Me refiero a quienes creen que las religiones son fuente de intolerancia, guerras y violencia y que, en consecuencia, un mundo sin religión alumbraría una especie de Arcadia feliz sin tensiones, animosidades, ni conflictos. Por el contrario, la pertenencia racionalmente crítica parte del convencimiento de la variada pluralidad de las identidades humanas y del hecho evidente de que todas ellas se entrecruzan 
sin que sea posible reducirlas uniformemente a una única e insalvable frontera, epígono, aunque se aparente lo contrario, de divisiones insuperables.

Ante la plural variedad de credos con los que se encuentra actualmente el Estado democrático ${ }^{4}$ y que aumentará en la medida en que continúe al desplazamiento e intercambio geográfico de personas de diversas y distintas procedencias geográficas, ideológicas y religiosas, quizás sea útil reiterar la referencia hecha a la historia del Cristianismo como pedagogía social y jurídica de la actitud que las autoridades estatales debieran asumir ante el fenómeno religioso y recordar lo que ya Hegel intuyó con histórica clarividencia al atribuir al Cristianismo la invención de la libertad como propiedad esencial y radical del ser humano, previa a cualquier concesión o atribución jurídica:

Esta idea [la de la libertad] llegó al mundo por obra del Cristianismo; para el cual el individuo (cada uno de los hombres en su singularidad) ha sido creado a imagen de Dios, y tiene valor infinito, y, siendo objeto y fin del amor de Dios, es destinado a tener una relación absoluta con Dios como Espíritu, hasta hacer que este Espíritu habite en él: el hombre, pues, está destinado en si mismo a la suma libertad (Hegel, $1969, \S 482)$.

\section{Referencias}

Andonegui, J. (1993). El desafío cristiano como desafio al cristianismo. En Lumen. Facultad de Teología de Vitoria. Año 42. 1993. Vitoria: Escuela Superior de Estudios Teológicos, 433-467.

Archi, G. (1976). Teodosio II e la sua codificazione. Primera Edición. Nápoles: Edizioni scientifiche italiane.

Bachelard, G. (1949). Le nouvel esprit scientifique. Primera Edición. París: PUF.

Barcellona, P. (2006). Critica della ragion laica. Colloqui con Michele Afferrante e Maurizio Ciampa, Primera Edición. Troina: Città Aperta.

Baubérot, J. (2001). La laïcisation et les mutations du religieux chrétien et du politique au XX $X^{\text {eme }}$ siècle. En Cristianesimo nella Storia. Istituto per le scienze religiose di Bologna. Año 22. 2001, Bologna: Ed. Dehoniane, pp. 633-657.

\footnotetext{
${ }^{4}$ Es evidente que el problema se agudiza en aquellas sociedades que se articulan en torno a modelos democráticos de gobierno, pues la carencia democrática es sinónimo de ausencia y negación del pluralismo.
} 
Berardino, A. (2002). La cristianizzazione del tempo nei secoli IV-V: la domenica. En Augustinianum. Institutum Patristicum “Augustinianum”. Año 42, número 1. 2002. Roma: Institutum Patristicum "Augustinianum”, pp. 97-125.

Bianchini, M. (1986). Cadenze liturgiche e calendario civile fra IV e V secolo. Alcune considerazioni. En Varios, Atti dei Convegni Internazionali dell'Accademia Romanistica Costantiniana. Perugia: Università degli studi. Volumen VI, pp. 241-263.

Braun, R. (1977). Deus Christianorum. Recherches sur le vocabulaire doctrinal du Tertulien. Segunda Edición. Paris: Études Augustiniennes.

Braun, R. (1964). Observations sur l'architecture de l'Apologeticum. En Renard, Marcel y Schilling, Robert (ed.). Hommages à Jean Bayet. Bruselas: Latomus, pp. 114-121.

Braun, R. (1979). Nouvelles observations linguistiques sur le rédacteur de la "Passio Perpetuae". En Vigiliae Christianae. Año 33, número 2. Brill, pp. 105-117.

Braun, R. (1972). Un nouveau Tertullien: problèmes de biographie et de chronologie. En Revue des Études Latines. Université de Paris IV - Sorbonne, Institut d'Études latines. Año 50, 1972. Paris: Société des Études Latines, pp. 67-84.

Bremmer, J., Formisano, M, (2012). (ed.), Perpetua's Passions. Multidisciplinary Approches to the Passio Perpetuae et Felicitatis. Primera Edición. Oxford-Nueva York: Oxford University Press.

Calvo, A. (1990). El Bautismo signo productor de la pertenencia a la Iglesia-una. En Scriptorium Victoriense. Facultad de Teología de Vitoria. Año 37. Vitoria: Escuela Superior de Estudios Teológicos, pp. 117-154.

Calvo, A. (1994). Para una aproximación al tópico "Derechos Humanos”. En Lumen. Facultad de Teología de Vitoria. Año 43. Vitoria: Escuela Superior de Estudios Teológicos, pp. 69-113.

Calvo, A. (2003). Paradojas del pluralismo. En Estudios de Deusto. Universidad de Deusto. Año 51, número 2, 2003. Bilbao: Publicaciones Universidad de Deusto, pp. 93-114.

Calvo, A. (2003). Pluralismo, multiculturalismo y tolerancia: semántica y derecho. En Scriptorium Victoriense. Facultad de Teología de Vitoria. Año 50. Vitoria: Escuela Superior de Estudios Teológicos, pp. 161-217.

Calvo, A. (2003). Tolerancia, multiculturalismo y democracia: límites de un problema. En Laicidad y libertades. Escritos jurídicos. Número 3. Madrid: Asociación "Derecho, Laicidad y Libertades", pp. 73-106.

Cochrane, Ch. (s. f.). Christianity and Classical Culture. Primera Edición. Nueva York: Liberty Fund.

Duguit, Ln. (1911). Le droit social, le droit individuel et la transformation de l'état: conférences faites à l'École des hautes études sociales. Primera Edición. Paris: Edit. F. Alcan.

Edward E. (2006). Black muslim religion in the Nation of Islam (1960-1975). The University of North Carolina Press. 
Ferrara, A. (1992). L'eudaimonia postmoderna: mutamento culturale e modelli di razionalità. Primera Edición. Nápoles: Liguori.

Frend, W. (1965). Martyrdom and Persecution in the Early Church: a study of a conflict from the Maccabees to Donatus. Primera Edición. Oxford: Blackwell.

Giardina, A. (1989). Le cose della guerra, Primera Edición. Milán: Fondazione Lorenzo Valla. Gigon, O. (1970). La cultura antigua y el Cristianismo, Primera Edición. Madrid: Gredos, 58. Guignebert, C. (1901). Tertulien. Études sur les sentiments à l'égard de l'Empire et de la société civile. Primera Edición. Paris: E. Leroux, 398 Gurevič, A. Kategorii srednevekovoj kul'tury, Izdatel'stvo «Iskusstvo», Moscú 1972. Se ha utilizado la versión italiana de esta obra traducida por C. Castelli y publicada en Turín el año 1983 por Giulio Einaudi bajo el título Le categorie della cultura medievale.

Gurevič, A. (1997). Los orígenes del individualismo europeo, Primera Edición. Barcelona: Editorial Crítica.

Habermehl, P. (2004). Perpetua Und Der Agypter Oder Bilder Des Bosen Im Fruhen Afrikanischen Christentum: Ein Versuch zur Passio Sanctarum Perpetuae et Felicitatis. Segunda Edición. Berlin-Nueva York; Walter De Gruyter Inc.

Hegel, G. (1969). Enzyklopädie derphilosophischen Wissenschaften im Grundisse (1830). Edición de F. Nicolin y Pöggeler. Hamburgo: Felix Meiner.

Holmes, S. (1994). Liberalism for a world of ethnic passions and decaying states. En Social research: An International Quarterly. Año 61, número 3, 1994. Arien Mack, pp. 599-610.

Hornus, J. (1960). Evangile et Labarum. Étude sur l'attitude du christianisme primitif devant les problémes de l'Etat, de la guerre et de la violence. Primera Edición. Ginebra: Labor et Fides.

Jiménez, J. (2003). "O amentia monstruosa!” A propósito de la cristianización de la liturgia imperial y del ritual circense durante el siglo $V$. En Cristianesimo nella Storia. Istituto per le scienze religiose di Bologna. Año 24, 2003, Bologna: Ed. Dehoniane, pp. 23-39.

Kateb, G. (1994). Notes on pluralism. En Social research: An International Quarterly. Año 61, número 3, 1994. Arien Mack, pp. 511-537.

Klein, R. (1968). Tertulianus und das römische Reich. Primera Edición. Heidelberg: Carl Winter. Universitaetsverlag.

Kukathas, C. (1992). Are there any cultural rights? En Political theory. Año 20, número 1, 1992. SAGE Publications, pp. 105-139.

Kymlicka, W. (1992). The rights of minority cultures. Reply to kathas. En Political theory. Año 20, número 1. SAGE Publications, pp. 140-146.

Kymlicka, W. (1995). Multicultural citizenship. A liberal theory of minority rights. Primera Edición. Oxford - Nueva York: Oxford University Press.

Lambin, R. (1999). Le voile des femmes. Un inventaire historique, social et psychologique. Primera Edición. Berlin, Nueva York, París, Viena: Peter Lang. 
Lazzati, G. (1956). Gli sviluppi della letteratura sui martiri nei primi quattro secoli. Primera Edición. Turín: Società editrice internazionale, 1956.

Lee, M. (1996). The Nation of Islam. An American Millenarian Movement. Primera Edición. Syracuse-Nueva York: Syracuse University Press.

Lincoln, Ch. (1994). The black muslims in America, Primera Edición. Grand Rapids-Michigan: Wm. B. Eerdmans Publishing Co.

Lombardi, G. (1986). Dall'Editto di Milano del 313 alla 'Dignitatis Humanae' del Vaticano II. En Studia et Documenta Historiae et Iuris. Pontificia Universitas Lateranensis. Año 52. Città del Vaticano: Lateran University Press, pp. 1-60.

Lombardi, G. (1984). L'editto di Milano del 313 e la laicità dello Stato. En Studia et Documenta Historiae et Iuris. Pontificia Universitas Lateranensis. Año 50. Città del Vaticano: Lateran University Press, pp. 1-98.

Llamazares, D. (2002). Derecho de la libertad de conciencia, vol. I. Libertad de conciencia y laicidad. Segunda Edición. Madrid: Civitas.

MacIntyre, A. (1995). Is patriotism a virtue?. En Beiner, R. (ed.), Theorizing Citizenship. Primera Edición. Albany: State University of New York Press, pp. 209-228.

Maffettone, S. (2006). Diritti umani e diversità culturale. En Sen, Amartya K., Fassino, Piero y Maffettone, Sebastiano (ed.). Giustizia globale, Primera Edición. Milán: Il Saggiatore, pp. 47-88.

Malaurie, P. (2003). Le droit et l'exigence de dignité. En Études. Volumen 398, n. 3985 .Paris: S.E.R. Publications, pp. 619-628.

Manaresi, A. (1914). L'Impero romano e il Cristianesimo, Primera Edición. Turín: Edit. Bocca. Margalit, A., Halbertal, M. (2004). Liberalism and the right to culture. En Social Research. An International Quarterly. Año 71, número 3. Arien Mack, pp. 529-548.

Mazzucco, C. (1975). Il significato della 'ibertas' proclamata dai martiri della "Passio Perpetuae", en Varios. Forma Futuri. Studi in onore del card. M. Pellegrino. Primera Edición. Turín: Bottega d'Erasmo, pp. 542-565.

Monceaux, P. (1901). Histoire littéraire de l'Afrique chrétienne depuis les origines jusqu' à l'invasion arabe, vol. I. Tertullien et les origines. Paris: Edit. Culture et civilisation.

Musurillo, H. (1972). The Acts of Christian Martyrs. Primera Edición. Oxford: Clarendon Press.

Nocera, G. (1983). Arte di governo e codificazione nel disegno di Teodosio II. En AARC. Volumen V.

Pace, E., Guolo, R. (2002). I fondamentalismi. Primera Edición. Roma-Bari: Laterza.

Pace, E. (2003). Politique de l'identité, Religion et enjeu scolaire. En Archives de Sciences sociales des Religions. École des hautes études en sciences sociales. Volumen 121. Paris: Éditions de l'École des hautes études en sciences sociales, pp. 115-126. 
Paolucci, G., Eid, C. (2006). I cristiani venuti dall'Islam. Storie di musulmani convertiti. Tercera Edición. Casale Monferrato (Alessandria): Piemme.

Paribeni, R. (1913). Sullorigine del nome Cristiano. En Nuovo Bulletino di Archeologia cristiana. Año 19. Roma: Libreria Spithover, pp. 37-41.

Pellegrino, M. (1947). Studi su l'antica apologetica. Primera Edición. Roma: Edizioni di "Storia e Letteratura".

Pellegrino, M. (1961). Le sens ecclésial du martyre. En Revue des sciences religieuses. Faculté de Théologie Catholique de Strasbourg. Año 35, Strasbourg: Faculté de Théologie Catholique, pp. 151-175.

Peretto, E. (1989). Clemente Romano ai Corinti. Sfida alla violenza. En Vetera Christianorum. Dipartimento di Studi classici e cristiani dell'Università di Bari. Año 26, 1989. Santo Spirito (Bari): Edipuglia srl, pp. 89-114.

Peterson, E. (1946). Christianus. En Miscellanea G. Mercati, I. Studi e Testi. Volumen 121. Ciudad del Vaticano, pp. 355-372.

Powell, D. (1975). Tertullianists and Cataphrygians. En VigiIiae Christianae. Año 29, 1975. Brill, pp. 33-54.

Puente, P. (1978). Tertuliano y el latín cristiano. Revisión de las diversas posiciones. En Durius. Universidad de Valladolid. Año 6, 1978. Valladolid: Departamento de Filología Latina, pp. 93-115.

Querejazu, J. (1994). Perplejidades de la tolerancia. En Lumen. Facultad de Teología de Vitoria. Año 43. Escuela Superior de Estudios Teológicos, pp. 42-57.

Rahner, H. (1990). Chiesa e struttura politica nel cristianesimo primitivo. Documenti della Chiesa nei primi otto secoli con introduzione e commento, tr. M. Morani y Giulia Regoliosi. Primera Edición. Milán: Jaca Book.

Rordorf, W. (1969). Tertulianus Beurteilung des Soldatenstandes. En Vigiliae Christianae. Año 23, 1969. Brill, pp. 105-141.

Sabbatucci, D. (1988). Sommario di storia delle religioni. Primera Edición. Roma: Bagatto Libri, 1988.

Saggioro, A. (1999). Dalla 'pompa diaboli' allo 'spiritale theatrum'. Cultura classica e cristianesimo nella polémica dei Padri della Chiesa contro gli spettacoli. Il terzo secolo (Mythos 8), Palermo.

Saggioro, A. (2003). Circus imago poli: la dimensione spazio-temporale dei ludi circenses, en Segarra Crespo, Diana (ed.). Transcurrir y Recorrer. La categoría espacio-temporal en las religiones del mundo clásico. Primera Edición. Roma-Madrid: CSIC, pp. 65-80.

Saggioro, A. (2003). Rapporti e conflitti tra paganesimo e cristianesimo nel Codice Teodosiano. En Annali di Storia dell'Esegesi. Università di Bologna. Año 20, número 1. Bologna: Centro Editoriale Dehoniano spa, pp. 165-181. 
Sartori, G. (2001). Pluralismo, Multiculturalismo e Estranei. Primera Edición. Milán: Rizzoli. Sen, A. (s. f.). Identità, povertà e diritti umani, en Sen, Amartya K., Fassino, Piero y Maffettone, Sebastiano (ed.). Giustizia globale, cit., pp. 13-32.

Sennett, R. 81997). The fall of public man: the forces eroding public life and burdening the modern psyche with roles it cannot perform. Primera Edición. Nueva York: Knopf.

Siniscalco, P. (1961). I martiri della Chiesa primitiva, en Varios, Martiri: Giudizio e Dono per la Chiesa. Primera Edición. Turín: Marietti, pp. 9-28.

Siniscalco, P. (2007). Il cammino di Cristo nellimpero romano. Sexta Edición. Roma- Bari: Laterza.

Sordi, Marta. (1957). I primi rapporti fra lo stato Romano e il Cristianesimo e l'origine delle persecuzioni. Primera Edición. Roma: Rendiconti Academia dei Lincei.

Sordi, M. (1964). L'Apología del martire romano Apollonio come fonte dell'Apologeticum di Tertuliano e i rapporti fra Tertuliano e Minucio Felice. En Rivista di Storia della Chiesa in Italia. Año 17. Roma: Fundación Michele Maccarrone, pp. 169-188.

Sordi, M. (2004). I Cristiani e l'Impero romano, Primera Edición reformada y actualizada. Milán: Jaca Book.

Staats, R. (1972). Ogdoas als Symbol für die Auferstehung. En Vigiliae Christianae. Año 26, 1972. Brill, pp. 29-52.

Staats, R. (1975). Die Sonntagnachtgottesdienste der christlichen Frübzeit. En Zeitschrift für die Neutestamentliche Wissenschaft und die Kunde der Älteren Kirche. Año 66, 1975. De Gruyter, pp. 242-263.

Taylor, Ch., Gutmann, A. (1992). (ed.). Multiculturalism and 'the politics of recognition': an essay. Primera Edición. Princeton: Princeton University Press.

Tertuliano. (1613). Apologeticus. Paris: P. Mettayer, p. 61.

Traquandi, G. (1973). Sulle presunte dipendenze dell'Apologeticum di Tertulliano dagli Atti di Apollonio. En Atene e Roma. Volumen 18, 1973. Società italiana per la diffusione e l'incoraggiamento degli studi classici, pp. 115-143.

Volterra, E. (1984). La costituzione introduttiva del Codice Teodosiano, en Varios, Sodalitas. Scritti in onore di A. Guarino, vol. VI. Primera Edición. Nápoles: Jovene-Giuffré, 3083-3103.

Waldron, J. (1992). Minority cultures and the cosmopolitan alternative. En University of Michigan journal of law reform. The University of Michigan. Volumen 01.25, 1992. Miller Canfield, pp. 751-793.

Zerbini, Z. (2003). Hic sunt leones: lo spazio e il tempo sacro delle venationes, en Segarra Crespo, Diana (ed.). Transcurrir y Recorrer. La categoria espacio-temporal en las religiones del mundo clásico. Primera Edición. Roma-Madrid: CSIC, pp. 81-90. 